\title{
HARMONIC MORPHISMS AND SUBHARMONIC FUNCTIONS
}

\author{
GUNDON CHOI AND GABJIN YUN
}

Received 18 July 2004 and in revised form 22 November 2004

Let $M$ be a complete Riemannian manifold and $N$ a complete noncompact Riemannian manifold. Let $\phi: M \rightarrow N$ be a surjective harmonic morphism. We prove that if $N$ admits a subharmonic function with finite Dirichlet integral which is not harmonic, and $\phi$ has finite energy, then $\phi$ is a constant map. Similarly, if $f$ is a subharmonic function on $N$ which is not harmonic and such that $|d f|$ is bounded, and if $\int_{M}|d \phi|<\infty$, then $\phi$ is a constant map. We also show that if $N^{m}(m \geq 3)$ has at least two ends of infinite volume satisfying the Sobolev inequality or positivity of the first eigenvalue of the Laplacian, then there are no nonconstant surjective harmonic morphisms with finite energy. For p-harmonic morphisms, similar results hold.

\section{Introduction}

Let $\left(M^{n}, g\right)$ and $\left(N^{m}, h\right)$ be complete Riemannian manifolds of dimension $n$ and $m$, respectively, and let $\phi: M \rightarrow N$ be a $C^{1}$ map. For a compact domain $D \subset M$, the energy $E$ of $\phi$ over $D$ is defined by

$$
E(\phi ; D)=\frac{1}{2} \int_{D}|d \phi|^{2} d v_{g}
$$

A map $\phi: M \rightarrow N$ is called harmonic if $\phi$ is a critical point of the energy functional defined by (1.1) on any compact domain $D \subset M$, or equivalently the tension field $\tau(\phi)=$ $\operatorname{tr}_{g} \nabla d \phi \in \Gamma\left(\phi^{-1} T N\right)$ is identically zero, where $\operatorname{tr}_{g}$ and $\nabla$ denote the trace with respect to the metric $g$ and Levi-Civita connection on $M$, respectively.

The classical Liouville theorem says that any bounded harmonic function defined on the whole plane must be a constant. Yau generalized [19] the Liouville theorem to harmonic functions on Riemannian manifolds of nonnegative Ricci curvature. Cheng [4] and Schoen and Yau [17] proved theorems of Liouville type for harmonic maps from a Riemannian manifold into a Riemannian manifold (see also [11]). In particular, Schoen and Yau proved that if $\phi: M \rightarrow N$ is a harmonic map from a complete, noncompact Riemannian manifold $M$ with nonnegative Ricci curvature to a complete Riemannian manifold $N$ with nonpositive sectional curvature with finite energy, then $\phi$ is constant. 
On the other hand, using the fact that the composition of a harmonic map and a convex function is subharmonic, Gordon proved [9] that every harmonic map from a compact Riemannian manifold to a Riemannian manifold which admits a strictly convex function is a constant map.

In [13], Kawai showed that if $M$ is a complete noncompact Riemannian manifold and $N$ is a Riemannian manifold having a $C^{2}$ strictly convex function $f: N \rightarrow \mathbb{R}$ such that the uniform norm $|d f|$ is bounded, then every harmonic map $\phi: M \rightarrow N$ with

$$
\int_{M}|d \phi|<\infty
$$

is a constant map.

In this paper, we consider harmonic morphisms between Riemannian manifolds and will prove similar results of Liouville type as mentioned above. The notion of harmonic morphism is stronger than harmonic map. In fact, it is known that every harmonic morphism is a harmonic map. Thus, it could be possible to replace the existence of a convex function by a weaker condition. Let $M$ be a complete Riemannian manifold and let $N$ be a complete noncompact Riemannian manifold admitting a subharmonic function $f$, but not harmonic (e.g., a strictly convex function). Let $\phi: M \rightarrow N$ be a surjective harmonic morphism. If either $|d f|$ is bounded and $\int_{M}|d \phi|<\infty$, or $|d f|$ is an $L^{2}$ function and $\phi$ has finite energy, then $\phi$ is a constant map. As a corollary, if $\phi: M \rightarrow N$ is a surjective harmonic morphism and $N$ is a simply connected Riemannian manifold of nonpositive sectional curvature, and if $\phi$ has finite energy or $\int_{M}|d \phi|<\infty$, then $\phi$ is a constant map. In case $\int_{M}|d \phi|<\infty$, the result is, in fact, due to Kawai [13].

On the other hand, any noncompact Riemannian manifold having at least two ends of infinite volume satisfying the Sobolev inequality or the positivity of the first eigenvalue of Laplacian admits a nonconstant bounded harmonic function with finite Dirichlet integral. Thus applying our main result to this, there are no nonconstant surjective harmonic morphisms from a Riemannian manifold onto such a manifold with at least two ends of infinite volume. In our results, we would like to remark that there is no kind of curvature conditions on $M$ comparing with other results or the main result in [5]. In [5], the authors proved that if $M$ is a complete Riemannian manifold with nonnegative Ricci curvature and $N$ is a complete Riemannian manifold with nonpositive scalar curvature, and if $\phi: M \rightarrow N$ is a harmonic morphism with finite energy, then $\phi$ is a constant map.

\section{Harmonic morphism and subharmonic functions}

A $C^{0}$ map $\phi: M \rightarrow N$ is called a harmonic morphism if for any harmonic function $f: U \rightarrow \mathbb{R}$ on an open set $U \subset N$ such that $\phi^{-1}(U)$ is nonempty, the composition $f \circ \phi: \phi^{-1}(U) \rightarrow \mathbb{R}$ is also a harmonic function on $\phi^{-1}(U)$. (Because of the existence of harmonic coordinates (cf. [10]), any harmonic morphism is necessarily $C^{\infty}$.)

Let $\phi:(M, g) \rightarrow(N, h)$ be a smooth map between Riemannian manifolds. Let $C_{\phi}:=$ $\left\{x \in M \mid d \phi_{x}=0\right\}$ be the critical set of $\phi$ and $M^{*}:=M-C_{\phi}$. At each point $p \in M^{*}$, the vertical space at $p$ is $V_{p}=\operatorname{ker} d \phi_{p} \subset T_{p} M$ and the horizontal space is $H_{p}=V_{p}^{\perp}$. A map $\phi:(M, g) \rightarrow(N, h)$ is said to be horizontally (weakly) conformal if there exists a function 
$\lambda: M^{*} \rightarrow \mathbb{R}^{+}$such that

$$
\lambda^{2} g(X, Y)=h(d \phi(X), d \phi(Y))
$$

for all $X, Y \in H_{p}$ and $p \in M^{*}$. Here $\lambda$ is called the dilation of $\phi$.

It is well known $[8,12]$ that a smooth map $\phi:(M, g) \rightarrow(N, h)$ between Riemannian manifolds is a harmonic morphism if and only if it is harmonic and horizontally weakly conformal. It is also well known that if $\operatorname{dim}(M)<\operatorname{dim}(N)$, then every harmonic morphism must be constant. Moreover, since any harmonic morphism is an open map, every harmonic morphism from a compact manifold into a noncompact manifold is a constant map.

We start with the following simple formula.

Lemma 2.1. Let $\phi: M \rightarrow N$ be a horizontally weakly conformal map between Riemannian manifolds of dimension $n$ and $m$, respectively, and $f: N \rightarrow \mathbb{R}$ be a $C^{2}$ function. Then for any $C^{1}$ function $\eta$ on $M$,

$$
\langle d(f \circ \phi), d \eta\rangle=-\frac{|d \phi|^{2}}{m} \eta(\Delta f) \circ \phi+\langle\nabla(\eta \cdot(d f) \circ \phi), d \phi\rangle .
$$

Proof. Let $\lambda$ be the dilation of $\phi$ and let $\left\{e_{i}\right\}_{i=1}^{n}$ be a local orthonormal frame which is normal at some point. If $\operatorname{dim}(M)=n<m=\operatorname{dim}(N)$, then $\phi$ is a constant and so (2.2) is obviously true. Thus, we may assume that $n \geq m$ and $d \phi\left(e_{j}\right)=0$ for $j \geq m+1$. Note that it follows from (2.1) that $\left\{E_{i}=(1 / \lambda) d \phi\left(e_{i}\right)\right\}$ is an orthonormal frame on $N$ where $\lambda \neq 0$ and

$$
m \lambda^{2}=|d \phi|^{2}
$$

Recall that the set $C_{\phi}$ at which $\lambda$ vanishes is discrete. One can compute (cf. [13])

$$
\langle\nabla(\eta \cdot(d f) \circ \phi), d \phi\rangle=\langle d(f \circ \phi), d \eta\rangle+\eta \sum_{i=1}^{m}\left\langle\nabla_{d \phi\left(e_{i}\right)}((d f) \circ \phi), d \phi\left(e_{i}\right)\right\rangle .
$$

Also it is easy to see from (2.3) that

$$
\left\langle\nabla_{d \phi\left(e_{i}\right)}(d f \circ \phi), d \phi\left(e_{i}\right)\right\rangle=\lambda^{2}\left\langle\nabla_{E_{i}}((d f) \circ \phi), E_{i}\right\rangle=\frac{|d \phi|^{2}}{m}(\Delta f) \circ \phi .
$$

Substituting (2.5) into (2.4), one obtains (2.2).

Proposition 2.2. Let $\phi: M \rightarrow N$ be a nonconstant surjective harmonic morphism between Riemannian manifolds. Assume $M$ is complete and noncompact. Let $f$ be a subharmonic function on N. Suppose that either

(i) $E(\phi)<\infty$ and $E(f)<\infty$, or

(ii) $\int_{M}|d \phi|<\infty$ and $|d f|$ is bounded.

Then $f$ is harmonic. 
Proof. Fix a point $p$ of $M$ and for $r>0$ choose a cut-off function $\eta$ with the following property:

$$
0 \leq \eta \leq 1, \quad|d \eta| \leq \frac{2}{r}, \quad \eta= \begin{cases}1 & \text { on } B_{p}(r), \\ 0 & \text { on } M-B_{p}(2 r),\end{cases}
$$

where $B_{p}(r)$ is the geodesic ball of radius $r$, centered at $p$. Using Lemma 2.1 together with harmonicity $\delta d \phi=0$, one obtains

$$
\frac{1}{m} \int_{M}|d \phi|^{2} \eta(\Delta f) \circ \phi=-\int_{M}\langle d(f \circ \phi), d \eta\rangle \leq \int_{M}|d \phi||d \eta|(|d f| \circ \phi) .
$$

In case (i), applying the Hölder inequality to (2.7),

$$
\int_{M}|d \phi||d \eta|(|d f| \circ \phi) \leq\left(\int_{M}(|d f| \circ \phi)^{2}\right)^{1 / 2}\left(\int_{M}|d \eta|^{2}|d \phi|^{2}\right)^{1 / 2} \leq \frac{C}{r} E(\phi) .
$$

In case (ii),

$$
\int_{M}|d \phi||d \eta||d f| \circ \phi \leq \frac{\tilde{C}}{r}
$$

In both cases, letting $r \rightarrow \infty$, one obtains from subharmonicity

$$
|d \phi|^{2}(\Delta f) \circ \phi=0
$$

Since $\phi$ is nonconstant and the points at which $d \phi=0$ are discrete, $\Delta f=0$ on $\phi(M)=N$.

Corollary 2.3. Let $M$ be a complete Riemannian manifold and $N$ a complete noncompact Riemannian manifold.

(1) If $N$ admits a subharmonic function $f$, but not harmonic such that $\int_{N}|d f|^{2}<\infty$, then there exist no nonconstant surjective harmonic morphisms $\phi: M \rightarrow N$ with $\int_{M}|d \phi|^{2}<\infty$.

(2) If $N$ admits a subharmonic function $f$, but not harmonic such that $|d f|$ is bounded, then there exist no nonconstant surjective harmonic morphisms $\phi: M \rightarrow N$ with $\int_{M}|d \phi|<\infty$.

Proof. If $M$ is compact, then any harmonic morphism $\phi: M \rightarrow N$ is constant since $\phi$ is an open map and $N$ is noncompact. In case $M$ is noncompact, the theorem follows from Proposition 2.2.

The existence of subharmonic functions is a much weaker condition than the existence of harmonic functions or convex functions. Proposition 2.2 shows that the existence or nonexistence of nonconstant surjective harmonic morphisms with finite energy depends on the topology of manifolds rather than the curvature conditions.

Theorem 2.4. Let $M$ be a complete Riemannian manifold and $N$ a simply connected Riemannian manifold with nonpositive sectional curvature. Then there exist no nonconstant surjective harmonic morphisms $\phi: M \rightarrow N$ with finite energy or $\int_{M}|d \phi|<\infty$. 
Proof. In case $M$ is compact, it is obvious and so we may assume that $M$ is noncompact. By Proposition 2.2, it suffices to show that $N$ has a strictly convex function $f$ whose $|d f|$ is bounded or a subharmonic function $f$ with finite energy, $\int_{N}|d f|^{2}<\infty$, and $\Delta f>0$ near a point. Since $N$ is simply connected and has nonpositive sectional curvature, it is well known that there exists a strictly convex function $f$ whose $|d f|$ is bounded $[2,13]$. In particular $f$ is not harmonic. Thus the proof follows directly from Proposition 2.2.

For the second case, fix a point $p \in N$ and consider the distance function $\rho(x)=$ $\operatorname{dist}(p, x)$. It is well known that $\rho$ is a convex function and smooth on $N-\{p\}$. Now for positive real numbers $\alpha>0$, and $\beta>0$ with $\alpha+\beta<1$, choose an increasing $C^{2}$ function $\xi:[0, \infty) \rightarrow \mathbb{R}$ so that $\xi \equiv 1$ on $[1, \infty)$ and $\xi(t)=\alpha t^{2}+\beta$ near $t=0$.

Define $f(x)=\xi \circ \rho(x)$. Then it is easy to see that

$$
\Delta f=\xi^{\prime \prime}(\rho)+\xi^{\prime}(\rho) \Delta \rho
$$

and so $f$ is a nonconstant subharmonic function. In particular, $\Delta f>0$ near $p$ and $\int_{N}|d f|^{2}<\infty$ since $\xi^{\prime}(t)=0$ for $t \geq 1$.

Let $D \subset N$ be a compact subset of $N$. An end $\mathscr{E}$ of $N$ with respect to $D$ is a connected unbounded component of $N \backslash D$. When we say that $\mathscr{E}$ is an end, it is implicitly assumed that $\mathscr{E}$ is an end with respect to some compact subset $D \subset N$. The monotonicity of the number of ends with respect to compact subsets allows us to define the number of ends of a manifold.

Now let $N$ be a complete Riemannian $m$-manifold with $m \geq 3$. If there is a constant $C_{s}>0$, depending only on $m$, such that for any $C^{2}$ function $\eta$ with compact support in $N$

$$
\left(\int_{N} \eta^{2 m /(m-2)}\right)^{(m-2) / m} \leq C_{s} \int_{N}|d \eta|^{2},
$$

we say $N$ satisfies the Sobolev inequality.

Theorem 2.5. Let $M$ be a complete Riemannian manifold and let $N$ be a complete noncompact Riemannian $m$-manifold $(m \geq 3)$ with at least two ends of infinite volume. Suppose that either

(i) the Sobolev inequality holds on $N$, or

(ii) the first eigenvalue $\lambda_{1}(N)$ of $N$ is positive.

Then there exist no nonconstant surjective harmonic morphisms $\phi: M \rightarrow N$ with finite energy.

Proof. It follows from [3] that there is a nonconstant bounded harmonic function $f$ on $N$ with $\int_{N}|d f|^{2}<\infty$. From boundedness we may assume $f \geq 1$ by adding some positive constant if necessary. Define $u=-\log f$ so that

$$
\Delta u=\frac{|d f|^{2}}{f^{2}} .
$$


Thus $u$ is a subharmonic function and

$$
\int_{N}|d u|^{2}=\int_{N} \frac{|d f|^{2}}{f^{2}} \leq \int_{N}|d f|^{2}<\infty .
$$

Moreover, since $f$ is not constant, $u$ is not harmonic. Consequently, it follows from Proposition 2.2 that there exist no nonconstant surjective harmonic morphisms $\phi: M \rightarrow$ $N$ with $\int_{M}|d \phi|^{2}<\infty$.

\section{3. $p$-harmonic morphisms}

A $C^{1}$ map $\phi: M \rightarrow N$ between Riemannian manifolds of dimension $n$ and $m$, respectively, is called a $p$-harmonic map $(p \geq 2)$ if it is a critical point of the $p$-energy functional

$$
E_{p}(\phi)=\int_{\Omega}|d \phi|^{p} d v_{g}
$$

for any bounded domain $\Omega \subset M$. It is well known $[1,16]$ that a $C^{2}$ map $\phi: M \rightarrow N$ is a $p$-harmonic map if and only if it satisfies the $p$-harmonic map equation

$$
\operatorname{tr}_{g}\left(\nabla\left(|d \phi|^{p-2} d \phi\right)\right)=\delta\left(|d \phi|^{p-2} d \phi\right)=0 .
$$

We call a 2-harmonic map just a harmonic map.

Note that the notion of $p$-harmonic map is a parallel generalization of harmonic map and some Liouville-type theorems for $p$-harmonic maps are known. For example, Takeuchi [18] proved that if $\phi: M \rightarrow N$ is a $p$-harmonic map from a complete noncompact Riemannian manifold $M$ of nonnegative Ricci curvature into a Riemannian manifold $N$ of nonpositive sectional curvature such that $E_{2 p-2}(\phi)<\infty$, then $\phi$ is a constant map. And Nakauchi [15] showed that if $E_{p}(\phi)<\infty$ with the same curvature conditions, then $\phi$ is constant. In [13], Kawai showed that if $M$ is a complete noncompact Riemannian manifold and $N$ is a Riemannian manifold having a $C^{2}$ strictly convex function $f: N \rightarrow \mathbb{R}$ such that $|d f|$ is bounded, then every $p$-harmonic map $\phi: M \rightarrow N$ with

$$
\int_{M}|d \phi|^{p-1}<\infty
$$

is a constant map. Note that any harmonic map or harmonic morphism is necessarily smooth because of the existence of harmonic coordinates (cf. [10]). However when $p \neq 2$, the degenerate ellipticity of (3.2) gives only $C^{1, \alpha}$-regularity even for minimizers of $p$ energy functional (3.1).

Definition 3.1. A map $\phi: M \rightarrow N$ is called a $p$-harmonic morphism if for any $p$-harmonic function $f: V \rightarrow \mathbb{R}$ defined on an open subset $V$ of $N$ with $\phi^{-1}(V)$ nonempty, the composition $f \circ \phi: \phi^{-1}(V) \rightarrow \mathbb{R}$ is also a $p$-harmonic function.

In [14], Loubeau characterized the $p$-harmonic morphisms as follows.

Theorem 3.2. A map $\phi: M \rightarrow N$ is called a p-harmonic morphism if and only if it is a horizontally weakly conformal and p-harmonic map. 
In [6], the authors proved that if $M$ is a complete noncompact Riemannian manifold of nonnegative Ricci curvature and $N$ is a Riemannian manifold of nonpositive scalar curvature, then any $p$-harmonic morphism of class $C_{\mathrm{loc}}^{1}$ such that $E_{p}(\phi)<\infty$ or $E_{2 p-2}(\phi)<\infty$ must be a constant.

In this section, using a similar identity as in Lemma 2.1, we will show that for $p$ harmonic morphisms, similar results as in Section 2 hold.

LeMmA 3.3. Let $\phi: M \rightarrow N$ be a horizontally weakly conformal map of $C_{\text {loc }}^{1}$ between Riemannian manifolds of dimension $n$ and $m$, respectively, and $f: N \rightarrow \mathbb{R}$ be a $C^{2}$ function. Then for any $C^{1}$ function $\eta$ on $M$,

$$
\left\langle|d \phi|^{p-2} d(f \circ \phi), d \eta\right\rangle=-\frac{|d \phi|^{p}}{m} \eta(\Delta f) \circ \phi+\left\langle\nabla(\eta \cdot(d f) \circ \phi),|d \phi|^{p-2} d \phi\right\rangle .
$$

Proof. Let $\lambda$ be the dilation of $\phi$ and let $\left\{e_{i}\right\}_{i=1}^{n}$ be an orthonormal frame which is normal at some point. We may assume that $n \geq m$ and $d \phi\left(e_{j}\right)=0$ for $j \geq m+1$. Then

$$
m \lambda^{2}=|d \phi|^{2}
$$

and note that it follows from $(2.1)$ that $\left\{E_{i}=(1 / \lambda) d \phi\left(e_{i}\right)\right\}$ is an orthonormal frame on $N$ where $\lambda \neq 0$. Recall that the set $C_{\phi}$ at which $\lambda$ vanishes is discrete. One can compute (cf. [13])

$$
\begin{aligned}
\left\langle\nabla(\eta \cdot(d f) \circ \phi),|d \phi|^{p-2} d \phi\right\rangle= & \left\langle|d \phi|^{p-2} d(f \circ \phi), d \eta\right\rangle \\
& +\eta|d \phi|^{p-2} \sum_{i=1}^{m}\left\langle\nabla_{d \phi\left(e_{i}\right)}((d f) \circ \phi), d \phi\left(e_{i}\right)\right\rangle .
\end{aligned}
$$

Since from (3.5)

$$
\left\langle\nabla_{d \phi\left(e_{i}\right)}(d f \circ \phi), d \phi\left(e_{i}\right)\right\rangle=\lambda^{2}\left\langle\nabla_{E_{i}}((d f) \circ \phi), E_{i}\right\rangle=\frac{1}{m}|d \phi|^{2}(\Delta f) \circ \phi,
$$

identity (3.4) follows from (3.6) and (3.7).

Proposition 3.4. Let $\phi: M \rightarrow N$ be a nonconstant surjective p-harmonic morphism of class $C_{\mathrm{loc}}^{1}$ between Riemannian manifolds. Assume $M$ is complete and noncompact. Let $f$ be a subharmonic function on $N$. Suppose that either

(i) $E_{2 p-2}(\phi)<\infty$ and $E(f)<\infty$, or

(ii) $E_{p-1}(\phi)<\infty$ and $|d f|$ is bounded.

Then $f$ is harmonic.

Proof. First of all, note that we may assume $\phi$ is of class $C_{\mathrm{loc}}^{3}$ from $[7,16]$. It follows from Lemma 3.3, subharmonicity, and the proof of Proposition 2.2 that

$$
|d \phi|^{p}(\Delta f) \circ \phi=0
$$

and so $f$ is harmonic on $N$. 
Corollary 3.5. Let $M$ be a complete Riemannian manifold and $N$ a complete noncompact Riemannian manifold.

(1) If $N$ admits a subharmonic function $f$, but not harmonic such that $\int_{N}|d f|^{2}<\infty$, then there exist no nonconstant surjective p-harmonic morphisms $\phi: M \rightarrow N$ with $\int_{M}|d \phi|^{2 p-2}<\infty$.

(2) If $N$ admits a subharmonic function $f$, but not harmonic such that $|d f|$ is bounded, then there exist no nonconstant surjective p-harmonic morphisms $\phi: M \rightarrow N$ with $\int_{M}|d \phi|^{p-1}<\infty$.

Theorem 3.6. Let $M$ be a complete Riemannian manifold and $N$ a simply connected Riemannian manifold with nonpositive sectional curvature. Then there exist no nonconstant surjective p-harmonic morphisms $\phi: M \rightarrow N$ with $E_{2 p-2}(\phi)<\infty$ or $E_{p-1}(\phi)<\infty$.

Proof. The proof follows from the proofs of Theorem 3.6 and Proposition 3.4.

Theorem 3.7. Let $M$ be a complete Riemannian manifold and let $N$ be a complete noncompact Riemannian $m$-manifold $(m \geq 3)$ with at least two ends of infinite volume. Suppose that either

(i) the Sobolev inequality holds on $N$, or

(ii) the first eigenvalue $\lambda_{1}(N)$ of $N$ is positive.

Then there exist no nonconstant surjective p-harmonic morphisms $\phi: M \rightarrow N$ with $E_{2 p-2}(\phi)<\infty$.

Proof. The proof is similar as that of Theorem 2.5.

\section{Acknowledgment}

The second author is supported by Grant no. R05-2000-000-00013-0(2002) from the Basic Research Program of the KOSEF.

\section{References}

[1] P. Baird and S. Gudmundsson, p-harmonic maps and minimal submanifolds, Math. Ann. 294 (1992), no. 4, 611-624.

[2] R. L. Bishop and B. O'Neill, Manifolds of negative curvature, Trans. Amer. Math. Soc. 145 (1969), 1-49.

[3] H.-D. Cao, Y. Shen, and S. Zhu, The structure of stable minimal hypersurfaces in $\mathbf{R}^{n+1}$, Math. Res. Lett. 4 (1997), no. 5, 637-644.

[4] S. Y. Cheng, Liouville theorem for harmonic maps, Geometry of the Laplace Operator (Univ. Hawaii, Honolulu, Hawaii, 1979), Proc. Sympos. Pure Math., vol. 36, American Mathematical Society, Rhode Island, 1980, pp. 147-151.

[5] G. Choi and G. Yun, A theorem of Liouville type for harmonic morphisms, Geom. Dedicata 84 (2001), no. 1-3, 179-182.

[6] A theorem of Liouville type for p-harmonic morphisms, Geom. Dedicata 101 (2003), $55-59$.

[7] F. Duzaar and M. Fuchs, On removable singularities of p-harmonic maps, Ann. Inst. H. Poincaré Anal. Non Linéaire 7 (1990), no. 5, 385-405.

[8] B. Fuglede, Harmonic morphisms between Riemannian manifolds, Ann. Inst. Fourier (Grenoble) 28 (1978), no. 2, vi, 107-144. 
[9] W. B. Gordon, Convex functions and harmonic maps, Proc. Amer. Math. Soc. 33 (1972), 433437.

[10] R. E. Greene and H. Wu, Embedding of open Riemannian manifolds by harmonic functions, Ann. Inst. Fourier (Grenoble) 25 (1975), no. 1, 215-235.

[11] S. Hildebrandt, Nonlinear elliptic systems and harmonic mappings, Proceedings of the 1980 Beijing Symposium on Differential Geometry and Differential Equations, Vols. 1, 2, 3, (Beijing, 1980), Science Press, Beijing, 1982, pp. 481-615.

[12] T. Ishihara, A mapping of Riemannian manifolds which preserves harmonic functions, J. Math. Kyoto Univ. 19 (1979), no. 2, 215-229.

[13] S. Kawai, p-harmonic maps and convex functions, Geom. Dedicata 74 (1999), no. 3, 261-265.

[14] E. Loubeau, On p-harmonic morphisms, Differential Geom. Appl. 12 (2000), no. 3, 219-229.

[15] N. Nakauchi, A Liouville type theorem for p-harmonic maps, Osaka J. Math. 35 (1998), no. 2, 303-312.

[16] N. Nakauchi and S. Takakuwa, A remark on p-harmonic maps, Nonlinear Anal. 25 (1995), no. 2, 169-185.

[17] R. Schoen and S. T. Yau, Harmonic maps and the topology of stable hypersurfaces and manifolds with non-negative Ricci curvature, Comment. Math. Helv. 51 (1976), no. 3, 333-341.

[18] H. Takeuchi, Stability and Liouville theorems of p-harmonic maps, Japan. J. Math. (N.S.) 17 (1991), no. 2, 317-332.

[19] S. T. Yau, Harmonic functions on complete Riemannian manifolds, Comm. Pure Appl. Math. 28 (1975), 201-228.

Gundon Choi: Global Analysis Research Center (GARC) and Department of Mathematical Sciences, Seoul National University, San 56-1, Shillim-Dong, Seoul 151-747, Korea

E-mail address: cgd@math.snu.ac.kr

Gabjin Yun: Department of Mathematics, Myongji University, San 38-2, Namdong, Yongin, Kyunggi-Do 449-728, Korea

E-mail address: gabjin@wh.myongji.ac.kr 


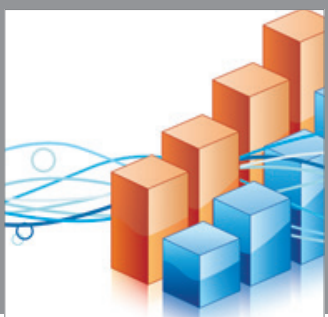

Advances in

Operations Research

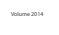

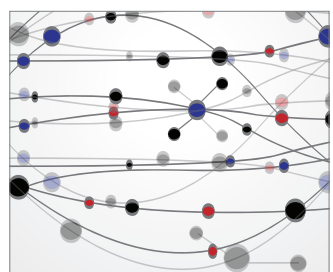

\section{The Scientific} World Journal
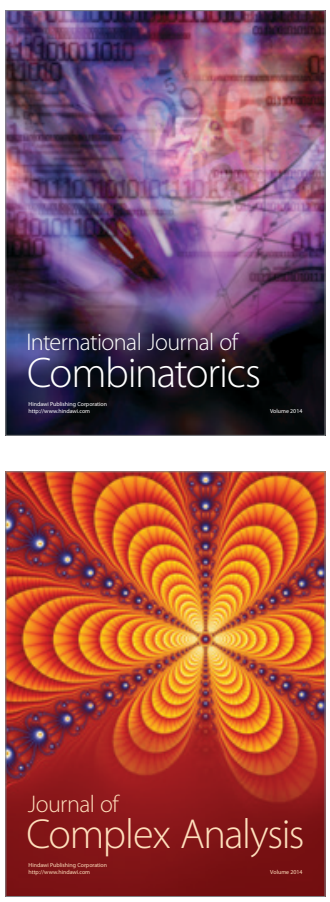

International Journal of

Mathematics and

Mathematical

Sciences
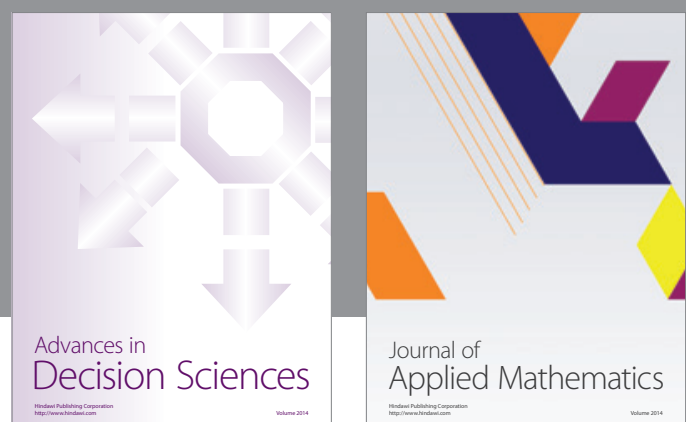

Journal of

Applied Mathematics
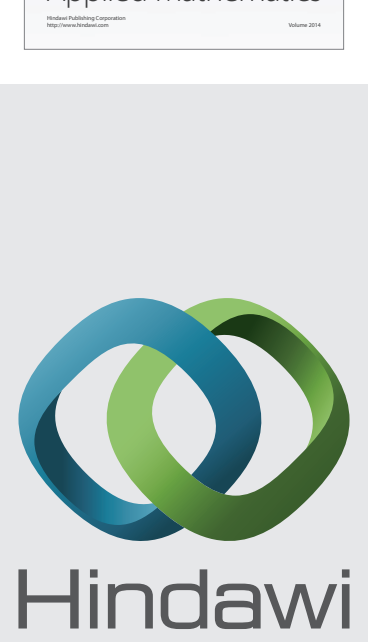

Submit your manuscripts at http://www.hindawi.com
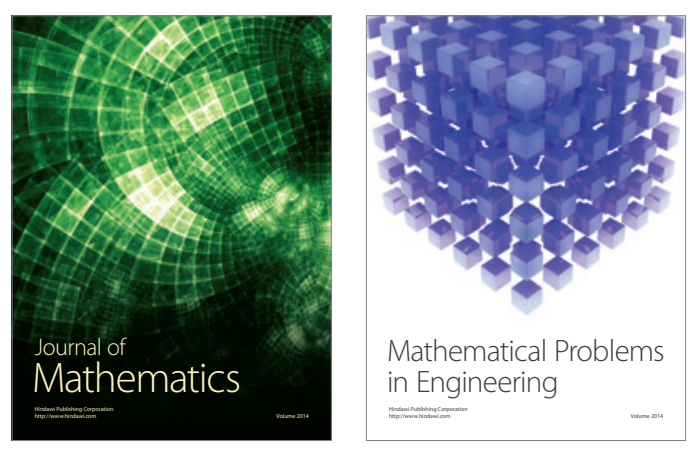

Mathematical Problems in Engineering
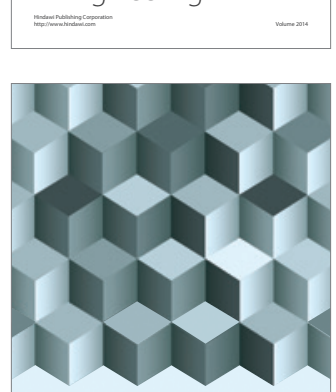

Journal of

Function Spaces
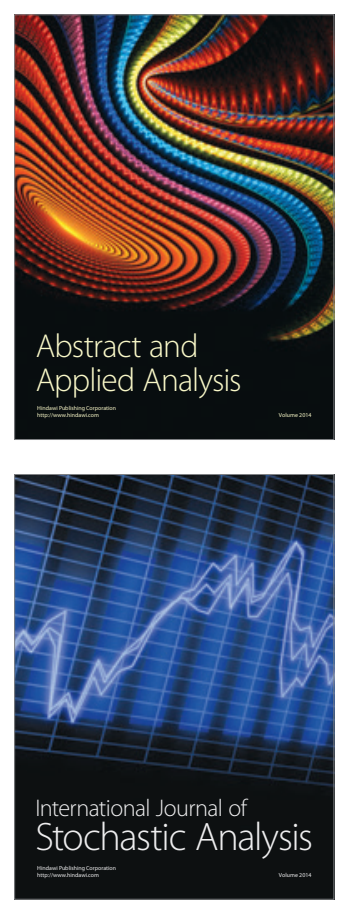

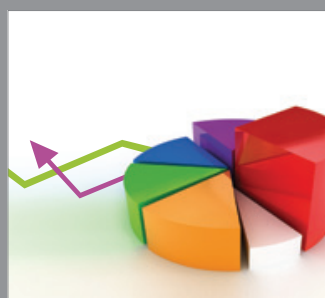

ournal of

Probability and Statistics

Promensencen
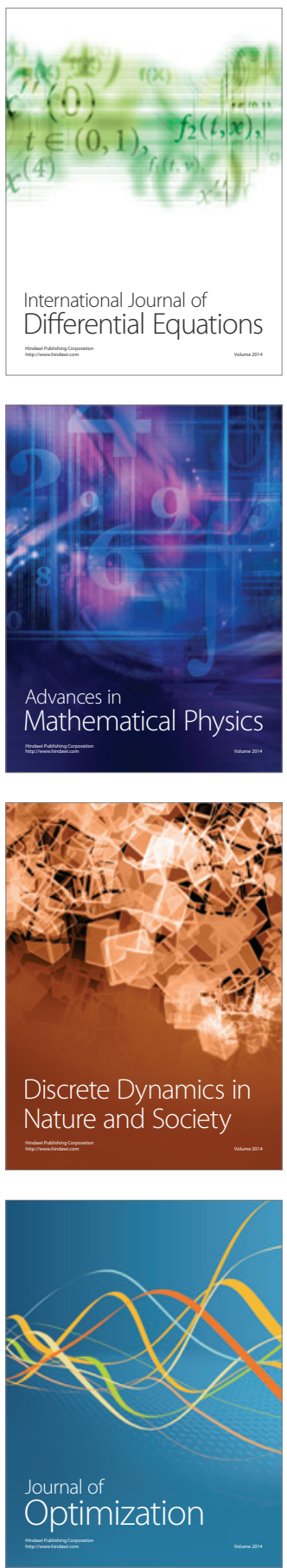